\title{
On combining partial and incompatible information in e-negotiation and e-arbitration
}

\author{
Josef Andrysek ${ }^{1}$, Antonella Bodini ${ }^{2}$, Miroslav Karny ${ }^{1}$, Jan Kracik ${ }^{1}$, \\ Fabrizio Ruggeri ${ }^{2}$ \\ ${ }^{1}$ UTIA, Academy of Sciences, Prague, Czech Republic, ${ }^{2}$ CNR-IMATI, \\ Milano, Italy
}

\begin{abstract}
The complexity of the problems to be addressed in an e-democracy framework and the variety of involved stakeholders, with different backgrounds, views and access to information sources, lead to consider the case in which enegotiation should be performed among subjects who have partial, sometimes incompatible, information and can hardly be gathered to discuss issues altogether, under the supervision of a facilitator. We propose a statistical method which addresses the issue of partial and incompatible information, merging it and then using it to get a final decision, possibly in an automatic way, through the processes of e-negotiation and e-arbitration.
\end{abstract}

AMS 2000 Subject Classification: Primary 62P25; Secondary 91B10.

Key Words: Frèchet class; Bayesian robustness; Kullback-Leibler divergence.

\section{Introduction}

Development of e-democracy practice is leading more and more towards situations in which larger communities are involved in decision processes, with stakeholders having different views and knowledge on the problems at hand. When problems are faced within a representative system by an elected body (parliament, city council, etc.), all the stakeholders have, more or less, access to the same information sources. In a statistical language, it means that different stakeholders are able to specify models on all the observable quantities (considered as random variables) and utilities. They can go even further, specifying prior beliefs on the parameters of model, via their prior distributions, according to the Bayesian paradigm. Negotiation and arbitration are therefore about how to combine different distributions and utilities defined on the same set of random quantities. Aggregation occurs either through consensus (negotiation) or through an external authoritative intervention (arbitration).

E-democracy should allow more people to be involved in the decision process, as in the project e21 about Local Agenda 21, recently approved by the Italian Ministry of Infrastructures and Innovation, which involves the Italian authors of the paper. In such project, citizens will be involved, directly or through representative groups, in informed discussions about environmental issues through mailing lists, forums, etc., with the possibility of accessing information via Internet. It is quite natural that nobody in such wide group will have access to all the information and that the stakeholders will not be able to specify models, priors and utilities on all the quantities. Therefore, the stakeholders will provide different distributions and utilities on different sets of random quantities. Suppose the case of a new military installation: the Army will have access to classified information, not available to the ordinary citizens, whereas scientists might have information on side effects (e.g. nuclear radiations) which might be neglected by the Army. In this scenario, the information can be not only partial, but also incompatible.

The process of negotiation to reach consensus is therefore quite difficult and the methods proposed in literature cannot be applied here, because, to our knowledge, they do not allow for handling incompatible distributions. Since the number of 
involved stakeholders is, in general, large, then any sound method should be made as automatic as possible and available for an easy use by a facilitator running the negotiation process. The heuristic method we present for combining probability distributions does not aim to solve the unsolvable problem of incompatible evidence; rather, it tries to identify its non controversial components in a way fulfilling the above requirements. To identify non controversial evidence and combine it, we extend an information-theoretic approach introduced in a simple case by Kracík (2004). Although our method could be applied to complex statistical models where both densities of observable quantities (models) and unknown parameters (priors) could be given via some partial, incompatible marginals distributions, here we will concentrate on some realistic scenarios in e-democracy, where stakeholders are able to specify probability density functions exactly, without any dependence upon parameters.

In Section 2 we will present the proposed approach, whereas its application in enegotiation and e-arbitration is discussed in Section 3. In Section 4 some examples are illustrated and few concluding remarks are presented in Section 5.

\section{The proposed method}

In Kracík (2004) it is suggested to combine probability density functions (pdf, for short) defined on different or partially overlapping spaces, so that the resulting pdf is close to each (partial) given pdf in some sense specified via the KullbackLeibler divergence. Instead of introducing cumbersome notations for the general formulation of the method, we will show it by a simple example.

Let $X_{1}, X_{2}$ be two random quantities and suppose that three experts provide the pdf's $f_{1}\left(x_{1}\right), f_{2}\left(x_{2}\right)$ and $f_{3}\left(x_{1}, x_{2}\right)$, respectively. Kracík (2004) defines a "compromise" among them as the pdf $\tilde{f}$ which minimizes

$$
\alpha_{1} K L\left(f_{1}\left(x_{1}\right), \tilde{f}\left(x_{1}\right)\right)+\alpha_{2} K L\left(f_{2}\left(x_{2}\right), \tilde{f}\left(x_{2}\right)\right)+\alpha_{3} K L\left(f_{3}\left(x_{1}, x_{2}\right), \tilde{f}\left(x_{1}, x_{2}\right)\right)
$$

where $\alpha_{i}$ are nonnegative, $\sum_{i} \alpha_{i}=1$, and $K L(p, q)$ is the Kullback-Leibler divergence of $p$ on $q$, defined as $\int p \log (p / q)$, (see for instance, Dacunha-Castelle and Duflo, 1986). Of course, we assume that all the necessary conditions for the finiteness of $K L$ are satisfied. It can be shown that $f$ is a solution of (1) if and only if it satisfies

$$
f\left(x_{1}, x_{2}\right)=\alpha_{1} f_{1}\left(x_{1}\right) f\left(x_{2} \mid x_{1}\right)+\alpha_{2} f_{2}\left(x_{2}\right) f\left(x_{1} \mid x_{2}\right)+\alpha_{3} f_{3}\left(x_{1}, x_{2}\right) .
$$

Assuming that each $f_{i}$ expresses the opinion of different stakeholders, we can interpret (2) by saying that each stakeholder completes his/her partial opinion by the corresponding part of the optimal "compromise" $\tilde{f}$. This interpretation is the starting point for the generalization introduced here.

Suppose $N$ random quantities $X_{1}, \ldots X_{N}$ are at stake and $K$ stakeholders are providing marginal pdf's on a (proper) subset of them. In particular, the $k$-th stakeholder will provide the pdf $f_{k}\left(X^{\left(I_{k}\right)}\right)$, where $X^{\left(I_{k}\right)}:=\left(X_{i_{1}}, \ldots, X_{i_{I_{k}}}\right)$ is a subset of $X^{(N)}:=\left(X_{1}, \ldots, X_{N}\right)$. This formulation allows for the extreme case of all marginal pdf's being undefined for some variable $X_{i}$ : we will not consider this case. It is worth mentioning that the marginal pdf's could be compatible and lead to a unique joint pdf, although we will be more interested in the cases in which either no compatible pdf exists or a class of compatible pdf's is obtained.

We assume that the $k$-th stakeholder has no opinions on the remaining $N-I_{k}$ random quantities so that any value taken by them will not affect his judgements about the variables in $X^{\left(I_{k}\right)}$. Formally, we translate this independence property into

$$
f_{k}\left(X_{(k)} \mid X\right) \equiv f_{k}\left(X_{(k)} \mid X \cap X^{\left(I_{k}\right)}\right)
$$


for any $X_{(k)} \subseteq X^{\left(I_{k}\right)}$ and any $X \subseteq X^{(N)}$, with $k=1, \ldots K$. Therefore the density is not changing if we add or remove a conditioning variable which is not in $X^{\left(I_{k}\right)}$.

We will use the property (3) in the next Step 2 as a building block for our combined pdf. In particular, we will be looking for the contribution of the $k$ th stakeholder to the conditional pdf $f\left(X_{n} \mid X_{n-1}, \ldots, X_{1}\right)$. If $X_{n} \notin X^{\left(I_{k}\right)}$, then no contribution is provided by the $k$-th stakeholder; otherwise, we introduce the notations $X_{n}^{\left(I_{k}\right)}:=X^{\left(I_{k}\right)} \cap\left\{X_{n+1}, \ldots, X_{N}\right\}$ and $Y_{n}^{\left(I_{k}\right)}:=X^{\left(I_{k}\right)} \cap\left\{X_{1}, \ldots, X_{n}\right\}$ to denote the random variables considered by the $k$-th stakeholder among, respectively, the last $N-n$ and the first $n$ elements of the ordering $X_{1}, \ldots, X_{N}$. Repeated applications of (3) will transform the middle term of the right hand side of

$$
f_{k}\left(X^{\left(I_{k}\right)}\right)=f_{k}\left(X_{n}^{\left(I_{k}\right)} \mid Y_{n}^{\left(I_{k}\right)}\right) f_{k}\left(X_{n} \mid Y_{n-1}^{\left(I_{k}\right)}\right) f_{k}\left(Y_{n-1}^{\left(I_{k}\right)}\right)
$$

into $f_{k}\left(X_{n} \mid X_{n-1}, \ldots, X_{1}\right)$, by adding the variables from $\left\{X_{n-1}, \ldots, X_{1}\right\}$ not considered by the $k$-th stakeholder. $f\left(\cdot \mid Y_{0}^{\left(I_{k}\right)}\right)$ has the meaning of $f(\cdot)$. In words, we have split the joint pdf into three parts using the chain's rule. The first involves a subset of $\left\{X_{N}, \ldots, X_{n+1}\right\}$, the second (the key one) is about $X_{n}$ and the third is about the variables $\left\{X_{n-1}, \ldots, X_{1}\right\}$ which are not considered by the stakeholder. Property (3) allows us to complement the second part to get a pdf on $X_{n}$ given all $X_{n-1}, \ldots, X_{1}$.

The basic method we propose to combine partial, incompatible information is described by the following

\section{Algorithm 1.}

1. Choose an ordering for the variables (e.g. $\left.X_{N}, \ldots, X_{1}\right)$ and apply the chain's rule, i.e.

$$
f\left(X_{1}, \ldots, X_{N}\right)=f\left(X_{N} \mid X_{N-1}, \ldots, X_{1}\right) \cdot f\left(X_{N-1} \mid X_{N-2} \ldots X_{1}\right) \cdot \ldots \cdot f\left(X_{1}\right) .
$$

2. For each component $f\left(X_{n} \mid X_{n-1}, \ldots, X_{1}\right), n=2, \ldots, N$, look for all the contributions of the stakeholders on it, i.e. either none or $f_{k}\left(X_{n} \mid X_{n-1}, \ldots, X_{1}\right)$. $f\left(X_{1}\right)$ is treated similarly.

3. Combine (linearly, with weights to be discussed later) all the contributions $f_{k}\left(X_{n} \mid X_{n-1}, \ldots, X_{1}\right)$ into $\widetilde{f}\left(X_{n} \mid X_{n-1}, \ldots, X_{1}\right)$, for $n=2, \ldots, N$. $\widetilde{f}\left(X_{1}\right)$ is treated similarly.

4. Get the joint density $\widetilde{f}\left(X_{1}, \ldots, X_{N}\right)$ via chain's rule, combining for $n=$ $2, \ldots, N$ all $\tilde{f}\left(X_{n} \mid X_{n-1}, \ldots, X_{1}\right)$ and $\tilde{f}\left(X_{1}\right)$, i.e.

$$
\tilde{f}\left(X_{1}, \ldots, X_{N}\right)=\tilde{f}\left(X_{N} \mid X_{N-1}, \ldots, X_{1}\right) \cdot \tilde{f}\left(X_{N-1} \mid X_{N-2} \ldots X_{1}\right) \cdot \ldots \cdot \widetilde{f}\left(X_{1}\right) .
$$

Since $\widetilde{f}$ depends on the selected order of the variables, a variant of the method considers the mixing over all $\widetilde{f}$ 's obtained for the $N$ ! possible permutations of the random quantities. We will not go into further details since it will add unnecessary complexity in the presentation and we deem that, in practical problems, stakeholders (or a facilitator in their behalf) could agree at least on the order.

\section{E-negotiation and e-arbitration}

The method presented in Section 2 (and illustrated later in Section 4 by some examples) can be applied in negotiations and arbitrations and, even better, in enegotiations and e-arbitrations. In fact, the former processes can be handled in 
physical meetings where facilitators can help stakeholders to find an agreement or discussions can be held so that an arbiter is aided in making a decision suitable for the stakeholders. Different techniques are used by the facilitators (or directly by the stakeholders themselves) in their effort of finding an agreement among the stakeholders, including mathematical ones, like, e.g., the approach based on nondominated actions described in Rios Insua, Holgado and Moreno (2004). In an e-participation environment, physical meetings are hardly possible since many stakeholders are involved at the same time and they might be scattered in very distant places. Therefore, there is the need for a (quite) automatic procedure on which the stakeholders agree. We will now present an approach, based on Algorithm 1, which can be seen as a first step towards this direction. For simplicity, we suppose that,

for all the involved random quantities $X_{i}$, there exists at least one stakeholder who provides an opinion, i.e. a marginal pdf, on it. Furthermore, we suppose that at least one stakeholder provides a marginal pdf different from the others, so that no complete agreement among stakeholders is possible.

Note that, from $(\mathrm{H})$ it follows that if all the stakeholders provide opinions on all the random quantities, then there is no partial knowledge but the pdf's are assumed to be incompatible. The procedure is described by the following steps. A few remarks will follow the description.

1. [Test for disjoint knowledge.] Check, by inspection of the stakeholders' marginal pdf's, if they provide opinions on disjoint subsets of random quantities $X_{i}$, forming a partition of them. If this is not the case, then go to Step 2. Otherwise, the marginal pdf's can be combined. If we assume them independent (i.e. property (3) holds), then there is a unique joint pdf which combines exactly the stakeholders' opinions: the product of the marginal pdf's. The procedure is then stopped. Otherwise, we consider the Frèchet class of all densities with marginal pdf's given by the stakeholders (see, e.g., Dall'Aglio, Kotz and Salinetti (1991) for more details on the Frèchet classes). The class is not empty since it contains, at least, the independent pdf. In this case, we move to Step 4.

2. [Test for incompatible knowledge.] Check, by inspection of the stakeholders' marginal pdf's if there is a joint pdf with such marginals. If this is not the case, go to Step 3. If such joint distribution exists and it is unique the procedure is stopped; otherwise we move to Step 4.

3. [Merging of partial or incompatible knowledge.] We apply Algorithm 1 to combine partial or incompatible marginals. We obtain a class of joint pdf's depending on the assigned weights (Step 3 of Algorithm 1) to each contribution. Then we move to Step 4.

4. [Test for robustness.] As discussed in the previous steps, we could obtain a class of joint pdf's by merging incompatible knowledge, by considering compatible marginals not leading to a unique joint pdf and disjoint knowledge leading to Frèchet classes. In all cases, a decision among the possible joint pdf's is needed: we suggest a quite automatic procedure which relies on the mathematical structure we gave to the problem. Let us suppose that the stakeholders can agree on some quantity of interest, summarizing the consequences of their choices (i.e., pdf's). If the range of this quantity is small, according 
to a shared criterion, then there is no need to look for a greater agreement among the stakeholders since the consequences of any choice of distributions in the class are quite irrelevant. We say that robustness is achieved and we stop the procedure. If the range is large, then the stakeholders can be asked to agree on a criterion to choose a pdf in the class: if this happens we move to Step 5; otherwise to Step 6.

5. [E-negotiation.] Once a class of pdf's is entertained, no robustness is achieved and the stakeholders agree on the necessity of a criterion to choose a pdf in the class, the role of the facilitator becomes fundamental. He/She will help the stakeholders in either reducing the width of the class of distributions, so that Steps 4 and 5 can be iterated, up to the achievement of a robust (sub)class; or in selecting a criterion to directly choose a pdf in the class.

6. [E-arbitration.] Once all the previous steps have failed and no agreed solution has been found, then an external authority (arbiter) is asked to make a decision, e.g. choosing the weights in Algorithm 1.

Summarizing the main features of our approach, which make it suitable for negotiation and arbitration over Internet in an e-democracy framework, we can say that

- the original opinions of the stakeholders are kept at any stage of the process;

- the opinions are combined with a very simple, objective rule;

- when considering Algorithm 1, facilitators and arbiters have to work only on the weights assigned to each opinion and they can be substituted, upon stakeholders' agreement, by automatic procedures to select a joint pdf.

Remark 1. Inspection of stakeholders' marginal pdf's to verify incompatibility can hardly show (and only in low dimensional problems) if there is no joint pdf with such marginals. A method to reduce the dimensionality of the problem is presented in Wang (2004). In any case, construction of a compatible joint pdf is, in general, a difficult task. A method, called Iterative Proportional Fitting (IFP), was proposed by Csiszár (1975). The IFP process is an iterative one which converges asymptotically to the sought joint pdf, if it exists. Of course, there is no way to tell if the joint density exists in general. A conjecture about detection of incompatibility via IFP was illustrated in Jiroušek and Vomlel (1994): cycling of IFP should be a symptom of incompatibility.

Remark 2. Of course, the facilitator could use his/her usual techniques to get an agreement among the stakeholders (Steps 4 and 5). The approach presented here stems from the statistical literature; in particular, is typical of Bayesian robustness and a plethora of methods are presented in Rios Insua and Ruggeri (2000).

Remark 3. It is worth mentioning that the direct choice of the weights arising from the application of Algorithm 1 and performed in Step 5, can be made by some automatic method. For instance, we can choose the values minimizing the sum of the squared distance between the $K$ stakeholders' marginals $f_{k}$ and the ones from the combined pdf $\tilde{f}$ for all possible values $x^{\left(I_{k}\right)}$ of the random variables $X^{\left(I_{k}\right)}$, i.e. in the discrete case,

$$
\sum_{k=1}^{K} \sum_{x^{\left(I_{k}\right)}}\left(f_{k}\left(x^{\left(I_{k}\right)}\right)-\tilde{f}\left(x^{\left(I_{k}\right)}\right)\right)^{2} .
$$


As an alternative, we could consider the minimization of

$$
\max _{k=1, K ; x^{\left(I_{k}\right)}}\left|f_{k}\left(x^{\left(I_{k}\right)}\right)-\widetilde{f}\left(x^{\left(I_{k}\right)}\right)\right| .
$$

In a robust Bayesian framework, another possibility is offered by the Gammaminimax approach: we will not illustrate this approach any further but we refer the interested reader to Ruggeri (2005).

\section{Examples}

We now illustrate how the proposed method works in some examples and, although they are mathematical ones by nature, we present them in a context of typical, but simple, problems in (e-)democracy.

\section{Example 1.}

Suppose that a new dam is proposed in an Italian mountain area where farming is the major activity of the inhabitants. The dam could be used to produce electricity, also for other parts of the country, and to start some industrial activity in the area. The effects on farming could be mixed: fields used for crops and cattle would be destroyed but a better irrigation system would benefit the remaining fields. There are three stakeholders involved in the process: local population (mostly farmers), the electricity company in charge of building the dam and the power plant and the geologists in charge of studying the effects of natural hazards on the dam (namely, earthquakes and landslides). On the top of them there is the regional government which is willing to find an agreement among the stakeholders and, in any case, has to take a decision about the construction of the dam.

We will concentrate just on one aspect of the problem: the assessment of the probabilities of the occurrence of some events. Therefore we will not consider utilities and we will not involve decision makers maximizing expected utilities. We identify three possible events: occurrence of a major earthquake (possible in most Italian areas), falling of a landslide in the artificial lake created by the dam (as it happened in Vajont, in North-East Italy in 1963 when 2,000 people died) and the benefits for the local economy.

The knowledge of the three stakeholders is different: the farmers have a strong opinion on the economical benefits and the past landslides, but they have no instruments to know about possible earthquakes. The electrical company has its opinion on the economical benefits and we suppose it can use its research center to make inference on possible earthquakes, based on available catalogues, but it cannot make any prediction about landslides. Finally, geologists can use their models to predict both earthquakes and landslides in the area but they have no instruments (and interest) in assessing about possible economical benefits.

We consider the following three binary variables:

- $X_{1}=\{$ Occurrence of a major landslide in the next 10 years $\}$ : no (0) or yes $(1)$

- $X_{2}=\{$ Positive benefit on the local economy in the next 10 years $\}$ : yes (0) or no (1).

- $X_{3}=\{$ Occurrence of a major earthquake in the next 10 years $\}$ : no (0) or yes $(1)$;

We denote farmers, electricity company and geologists with labels 1,2 and 3, respectively; their pdf's are therefore denoted as $f_{1}, f_{2}$ and $f_{3}$. As mentioned before, 
all of them are able to give only (marginal) densities on two random quantities, as shown in Table 1, coming from Wang (2004) who shows that there exists no joint pdf of three random variables with the given marginal densities.

Table 1: Values of marginal densities on their support

\begin{tabular}{|c|c|c|c|c|}
\hline Marginal & $(0,0)$ & $(0,1)$ & $(1,0)$ & $(1,1)$ \\
\hline$f_{1}\left(x_{1}, x_{2}\right)$ & 0.47 & 0.13 & 0.13 & 0.27 \\
$f_{2}\left(x_{2}, x_{3}\right)$ & 0.47 & 0.13 & 0.13 & 0.27 \\
$f_{3}\left(x_{1}, x_{3}\right)$ & 0.30 & 0.30 & 0.30 & 0.10 \\
\hline
\end{tabular}

Therefore, the farmers think that there is a $47 \%$ chance of no landslide and positive economical benefits; the geologists think of a $10 \%$ chance of both earthquake and landslide occurrences, and so on.

The three variables $X_{1}, X_{2}$ and $X_{3}$ have a Bernoulli distribution with probability 0.6 of being equal to 0 ; it means that farmers and electricity company agree on a $60 \%$ chance of positive economical benefits, and so on.

We use the method proposed in the previous sections to combine the evidence provided by the three incompatible marginals.

We apply now Algorithm 1 described in Section 2 and suppose that three stakeholders agree on the order of the chain's rule, so that they consider (Step 1)

$$
f\left(x_{1}, x_{2}, x_{3}\right)=f\left(x_{3} \mid x_{2}, x_{1}\right) f\left(x_{2} \mid x_{1}\right) f\left(x_{1}\right) .
$$

As in Step 2, we split first the three given marginals $f_{1}, f_{2}$ and $f_{3}$ following the same order of (4), i.e.

- $f_{1}\left(x_{1}, x_{2}\right)=f_{1}\left(x_{2} \mid x_{1}\right) f_{1}\left(x_{1}\right)$

- $f_{2}\left(x_{2}, x_{3}\right)=f_{2}\left(x_{3} \mid x_{2}\right) f_{2}\left(x_{2}\right)$

- $f_{3}\left(x_{1}, x_{3}\right)=f_{3}\left(x_{3} \mid x_{1}\right) f_{3}\left(x_{1}\right)$,

and then we complete the densities using the independence property (3), obtaining

- $f_{1}\left(x_{1}, x_{2}\right)=f_{1}\left(x_{2} \mid x_{1}\right) f_{1}\left(x_{1}\right)$

- $f_{2}\left(x_{2}, x_{3}\right)=f_{2}\left(x_{3} \mid x_{2}, x_{1}\right) f_{2}\left(x_{2} \mid x_{1}\right)$

- $f_{3}\left(x_{1}, x_{3}\right)=f_{3}\left(x_{3} \mid x_{2}, x_{1}\right) f_{3}\left(x_{1}\right)$.

In the example at hand, $f_{1}\left(x_{1}\right)$ and $f_{3}\left(x_{1}\right)$ contribute to $f\left(x_{1}\right)$, whereas $f_{1}\left(x_{2} \mid x_{1}\right)$ and $f_{2}\left(x_{2}\right)$ contribute to $f\left(x_{2} \mid x_{1}\right)$ and $f_{2}\left(x_{3} \mid x_{2}\right)$ and $f_{3}\left(x_{3} \mid x_{1}\right)$ contribute to $f\left(x_{3} \mid x_{2}, x_{1}\right)$. We combine linearly these probabilities (Step 3), assigning weights $\alpha, \beta$ and $\gamma$, respectively. Summarizing, we get

$$
\begin{aligned}
f\left(x_{1}\right) & =\alpha f_{1}\left(x_{1}\right)+(1-\alpha) f_{3}\left(x_{1}\right) \\
f\left(x_{2} \mid x_{1}\right) & =\beta f_{1}\left(x_{2} \mid x_{1}\right)+(1-\beta) f_{2}\left(x_{2}\right) \\
f\left(x_{3} \mid x_{2}, x_{1}\right) & =\gamma f_{2}\left(x_{3} \mid x_{2}\right)+(1-\gamma) f_{3}\left(x_{3} \mid x_{1}\right) .
\end{aligned}
$$

It is worth mentioning that we can omit $\alpha$ since $f_{1}\left(x_{1}\right) \equiv f_{3}\left(x_{1}\right)$. Applying the chain's rule to (5), we obtain (Step 4) the joint pdf $f$ (for simplicity, we use $f$ instead of $\widetilde{f})$, given by Table 2 .

We observe that the $f\left(x_{1}\right), f\left(x_{2}\right)$ and $f\left(x_{3}\right)$ are Bernoulli marginals which assign probability 0.6 to the outcome 0 , whereas the pairwise marginals are given in Table 3 .

The stakeholders and the regional government could use these ranges of probabilities to compute expected utilities (provided they agree on the same utility 
Table 2: Values of $f\left(x_{1}, x_{2}, x_{3}\right)$ on its support

\begin{tabular}{|c|c|}
\hline$\left(x_{1}, x_{2}, x_{3}\right)$ & $f\left(x_{1}, x_{2}, x_{3}\right)$ \\
\hline$(0,0,0)$ & $(30+17 \gamma)(36+11 \beta) / 6000$ \\
$(0,0,1)$ & $(30-17 \gamma)(36+11 \beta) / 6000$ \\
$(0,1,0)$ & $(20-7 \gamma)(24-11 \beta) / 4000$ \\
$(0,1,1)$ & $(20+7 \gamma)(24-11 \beta) / 4000$ \\
$(1,0,0)$ & $(45+2 \gamma)(24-11 \beta) / 6000$ \\
$(1,0,1)$ & $(15-2 \gamma)(24-11 \beta) / 6000$ \\
$(1,1,0)$ & $(30-17 \gamma)(16+11 \beta) / 4000$ \\
$(1,1,1)$ & $(10+17 \gamma)(16+11 \beta) / 4000$ \\
\hline
\end{tabular}

Table 3: Values of the bivariate marginals of $f$ on its support

\begin{tabular}{|c|c|c|}
\hline & $(0,0)$ & $(0,1)$ \\
\hline$f\left(x_{1}, x_{2}\right)$ & $0.36+0.11 \beta$ & $0.24-0.11 \beta$ \\
$f\left(x_{1}, x_{3}\right)$ & $0.3+(0.06+0.05 \beta) \gamma$ & $0.3-(0.06+0.05 \beta) \gamma$ \\
$f\left(x_{2}, x_{3}\right)$ & $0.36-(0.0275-0.0275 \gamma) \beta+0.11 \gamma$ & $0.24+(0.0275-0.0275 \gamma) \beta-0.11 \gamma$ \\
\hline \hline & $(1,0)$ & $(1,1)$ \\
\hline$f\left(x_{1}, x_{2}\right)$ & $0.24-0.11 \beta$ & $0.16+0.11 \beta$ \\
$f\left(x_{1}, x_{3}\right)$ & $0.3-(0.06+0.05 \beta) \gamma$ & $0.1+(0.06+0.05 \beta) \gamma$ \\
$f\left(x_{2}, x_{3}\right)$ & $0.24+(0.0275-0.0275 \gamma) \beta-0.11 \gamma$ & $0.16-(0.0275-0.0275 \gamma) \beta+0.11 \gamma$ \\
\hline
\end{tabular}

functions) and study the effect of the choice of $\beta$ and $\gamma$ on the maximum expected utility. We will not pursue this approach any further.

At this point the stakeholders could agree on the weights $\beta$ and $\gamma$ or, in absence of an agreement, the regional government could decide how to attribute the weights.

Note that $f\left(x_{1}, x_{2}\right)$ and $f_{1}\left(x_{1}, x_{2}\right)$ coincide if and only if $\beta=1$; if $\gamma=0$ then $f\left(x_{1}, x_{3}\right)$ and $f_{3}\left(x_{1}, x_{2}\right)$ coincide too and, in this case, $f\left(x_{1}, x_{2}\right)$ and $f_{2}\left(x_{1}, x_{2}\right)$ do not coincide, as shown in Table 4.

Table $4: \beta=1, \gamma=0$

\begin{tabular}{|l|l|l|l|l|}
\hline & $(0,0)$ & $(0,1)$ & $(1,0)$ & $(1,1)$ \\
\hline$f_{2}\left(x_{2}, x_{3}\right)$ & .47 & .13 & .13 & .27 \\
$f\left(x_{2}, x_{3}\right)$ & .3325 & .2675 & .2675 & .1325 \\
\hline
\end{tabular}

Finally, in Table 5 we show the democratic case, i.e. uniform weights.

Table 5 shows that the stakeholders' marginal pdf's are very close to the ones determined by the joint pdf obtained with Algorithm 1. Closer values could be obtained by considering the automatic procedures described in Remark 3 of Section 3.

\section{Example 2.}

A new organization of the work is introduced in a company and its effects on a period of 10 years are discussed by three stakeholders: the leaders of two different trade unions and the company's CEO, labeled 1,2 and 3, respectively. The effects are measured as the increase in number of employees $\left(X_{1}\right)$, the decrease in average salary, adjusted for inflation $\left(X_{2}\right)$ and the increase in company's profits $\left(X_{3}\right)$. The stakeholders agree in normalizing the random quantities, subtracting their means and dividing by their standard deviations, so that $X_{i} \sim \mathcal{N}_{1}(0,1)$ for $i=1,2,3$. They provide the following bivariate Gaussian pdf's:

$$
f_{1}\left(x_{1}, x_{2}\right)=\mathcal{N}_{2}\left(0, \Sigma_{1}\right), \quad f_{2}\left(x_{1}, x_{2}\right)=\mathcal{N}_{2}\left(0, \Sigma_{2}\right), \quad f_{3}\left(x_{2}, x_{3}\right)=\mathcal{N}_{2}\left(0, \Sigma_{3}\right)
$$


Table 5: $\beta=0.5, \gamma=0.5$

\begin{tabular}{|l|l|l|l|l|}
\hline & $(0,0)$ & $(0,1)$ & $(1,0)$ & $(1,1)$ \\
\hline$f_{1}\left(x_{1}, x_{2}\right)$ & .47 & .13 & .13 & .27 \\
$f\left(x_{1}, x_{2}\right)$ & .415 & .185 & .185 & .215 \\
\hline \hline$f_{2}\left(x_{2}, x_{3}\right)$ & .47 & .13 & .13 & .27 \\
$f\left(x_{2}, x_{3}\right)$ & .408125 & .191875 & .191875 & .208125 \\
\hline \hline$f_{3}\left(x_{1}, x_{3}\right)$ & .30 & .30 & .30 & .10 \\
$f\left(x_{1}, x_{3}\right)$ & .3425 & .2575 & .2575 & .1425 \\
\hline
\end{tabular}

where

$$
\Sigma_{1}=\left(\begin{array}{ll}
1 & 0 \\
0 & 1
\end{array}\right) \quad \Sigma_{2}=\left(\begin{array}{cc}
1 & 0.5 \\
0.5 & 1
\end{array}\right) \quad \Sigma_{3}=\left(\begin{array}{cc}
1 & 0.9 \\
0.9 & 1
\end{array}\right)
$$

are the variance-covariance matrices. The major difference is about the opinions of the trade unions leaders about the increase of manpower and the decrease in average salary: the first thinks that there is no correlation among them whereas the second believes in a quite strong, positive correlation. Following our approach we have that

$$
\begin{gathered}
f\left(x_{1}\right)=\alpha f_{1}\left(x_{1}\right)+(1-\alpha) f_{2}\left(x_{1}\right)=\mathcal{N}(0,1) \\
f\left(x_{2} \mid x_{1}\right)=\beta_{1} f_{1}\left(x_{2} \mid x_{1}\right)+\beta_{2} f_{2}\left(x_{2} \mid x_{1}\right)+\beta_{3} f_{3}\left(x_{2}\right)=\beta \mathcal{N}\left(\frac{1}{2} x_{1}, \frac{3}{4}\right)+(1-\beta) \mathcal{N}(0,1) \\
f\left(x_{3} \mid x_{2}, x_{1}\right)=f_{3}\left(x_{3} \mid x_{2}\right)=\mathcal{N}\left(\frac{9}{10} x_{2}, \frac{19}{100}\right)
\end{gathered}
$$

and, by the chain rule, $f$ turns out to be mixture of two multivariate Gaussian distribution with null mean and variance-covariance matrix:

$$
\left(\begin{array}{ccc}
1 & 0.50 & 0.45 \\
0.50 & 1 & 0.90 \\
0.45 & 0.90 & 1
\end{array}\right) \quad \text { and } \quad\left(\begin{array}{ccc}
1 & 0 & 0 \\
0 & 1 & 0.9 \\
0 & 0.9 & 1
\end{array}\right)
$$

respectively. Note that, although no stakeholder has provided a joint opinion on $X_{1}$ and $X_{3}$, a correlation has been induced by the other opinions as evidenced by the first covariance matrix which assigns a correlation 0.45 between $X_{1}$ and $X_{3}$.

\section{Discussion}

We have formulated a problem in which partial and incompatible opinions from stakeholders are expressed by pdf's and we have proposed a procedure, statistically driven, which could be applied in e-negotiation and e-arbitration. Some examples have shown its practical application. The proposed method allows in general to obtain proper pdf's which are "close" to the stakeholders' marginals, except for very pathological cases in which Dirac measures are involved, as in the following

\section{Example 3.}

Consider the following pdf's: $f_{2}\left(x_{1}\right) \equiv \operatorname{Bernoulli}(c) ; f_{3}\left(x_{3}\right) \equiv \operatorname{Bernoulli}(z)$; $f_{1}\left(x_{1}, x_{2}\right)$ as follows:

\begin{tabular}{|c|c|c|c|c|}
\hline & $(0,0)$ & $(0,1)$ & $(1,0)$ & $(1,1)$ \\
\hline$f_{1}\left(x_{1}, x_{2}\right)$ & 0 & 0 & $1-a$ & $a$ \\
\hline
\end{tabular}

thus $f_{1}\left(x_{1}\right) \equiv \delta_{1}$, the Dirac distribution concentrated on 1 , and $f_{1}\left(x_{2}\right) \equiv \operatorname{Bernoulli}(a)$. According to the approach previously described we should define

$$
f\left(x_{1}, x_{2}, x_{3}\right)=f_{3}\left(x_{3}\right) f_{1}\left(x_{2} \mid x_{1}\right)\left(\lambda f_{1}\left(x_{1}\right)+(1-\lambda) f_{2}\left(x_{1}\right)\right) .
$$


However $f_{1}\left(x_{2} \mid x_{1}\right)$ is not well defined. The interest of this example mainly lies in the fact that if $c$ is close to 1 then $f_{1}\left(x_{1}\right)$ and $f_{2}\left(x_{1}\right)$ are "quite compatible": nevertheless their composition in the indicated way is impossible, without some suitable conditions.

The proposed method is heuristically justified but current research aims to justify its use as the optimal procedure according to a suitable criterion. The main effort is about the extension of the result in Kracík (2004) on the optimality with respect to the Kullback-Leibler divergence.

\section{Acknowledgements}

This work was supported by the CNR - AV ČR grant on Advanced techniques of Bayesian decision making in complex systems and the European Science Foundation programme Towards Electronic Democracy: an Internet based decision support system. The authors wish to thank Radim Jiroušek and Jiří Vomlel for introducing them to the Iterative Proportional Fitting procedure.

\section{References}

Csiszár, I. (1975). I-divergence geometry of probability distribution and minimization problems, Ann. Prob., 3,146-158.

Dacunha-Castelle, D., and Duflo, M. (1986). Probability and Statistics I. Springer, New York.

Dall'Aglio, G., Kotz, S. and Salinetti, G. Eds. (1991). Advances in Probability Distributions with Given Marginals, Kluwer, Dordrecht.

Kracík, J. (2004). On composition of probability density function. In Multiple Participant Decision Making (Andrýsek, J., Kárný, M. and Kracík, J. eds.), 113-121. Advanced Knowledge International, Adelaide, Australia.

Jiroušek, R. and Vomlel, J. (1994). Inconsistent knowledge integration in a probabilistic model. In Proceedings of the Workshop on Mathematical Models for Handling Partial Knowledge in Artificial Intelligence, 263-270, Plenum Publ. Corp., Erice, Italy.

Rios Insua, D., Holgado, J. and Moreno, R. (2004). Multicriteria e-negotiation systems in e-democracy. Tech. Report, Universidad Rey Juan Carlos, Mostoles, Spain.

Rios Insua, D., and Ruggeri, F. (eds.) (2000). Robust Bayesian Analysis, SpringerVerlag, New York.

Ruggeri, F. (2005). Gamma-minimax inference. To appear in Encyclopedia of Statistical Sciences, 2nd ed., (Balakrishnan, N., Read, C.B. and Vidakovic, B. eds.), Wiley, New York, USA.

Wang, Y.J. (2004). Compatibility among marginal densities, Biometrika, 91, 234239. 